\title{
A Molecular Dynamics Study on the Miscibility of Polyglycolide with Different Polymers
}

\author{
Mahamat Bichara Abderaman, 3, *, El-Hadji Oumar Gueye ${ }^{1}$, Abdoulaye Ndiaye Dione ${ }^{1}$, \\ Alioune Aidara Diouf ${ }^{1}$, Omar Faye ${ }^{1,2}$, Aboubaker Chedikh Beye ${ }^{1}$ \\ ${ }^{1}$ Department of Physics, Faculty of Science and Technics, Cheikh Anta Diop University, Dakar, Senegal \\ ${ }^{2}$ Department of Mechanical Engineering and College of Engineering, University of Saskatchewan, Saskatchewan, Canada \\ ${ }^{3}$ Departement of Chemical Engineering, Higher National Petroleum Institute of Mao, Mao, Chad
}

\section{Email address:}

tindy1979@yahoo.fr (Mahamat B. A.), elhadjioumar.gueye@gemail.com (El-Hadji O. G.), acbeye@gemail.com (Aboubaker C. B.), omsofaye@yahoo.fr (Omar F.), bayfalldione@yahoo.fr (Abdoulaye N. D.), aliounedioufaidara@hotmail.fr (Alioune A. D.)

${ }^{*}$ Corresponding author

\section{To cite this article:}

Mahamat Bichara Abderaman, El-Hadji Oumar Gueye, Abdoulaye Ndiaye Dione, Alioune Aidara Diouf, Omar Faye, Aboubaker Chedikh Beye. A Molecular Dynamics Study on the Miscibility of Polyglycolide with Different Polymers. International Journal of Materials Science and Applications. Vol. 7, No. 4, 2018, pp. 126-132. doi: 10.11648/j.ijmsa.20180704.12

Received: April 30, 2018; Accepted: May 31, 2018; Published: June 13, 2018

\begin{abstract}
The miscibility of mixtures between polyglycolide and the following polymers: polyethylene, polystyrene, polyacrylonitrile and polylactide is studied by Molecular Dynamics Simulation using Forcite and Blends Modules. The simulations of the binary mixture for the evaluation of the energy is achieved in the framework of the Flory-Huggins model. The Flory-Huggins interaction parameter, the mixing energy and the phase diagrams are analyzed and found to be the main parameters and features controlling the miscibility process in the present computer simulations. The results of the simulation show that when the Flory-Huggins interaction parameter Chi to a value close to 1 of mixtures the polyglycolide / polylactide, polyglycolide / polyacrylonitrile, polyglycolide / polyethylene and polyglycolide / polystyrene are miscible at 50K, $230 \mathrm{~K}$, $238 \mathrm{~K}$ and $378 \mathrm{~K}$ respectively. The commonly-accepted miscibility criteria of the binary namely mixing when Chi is negative or positive but small and non-miscibility when Chi is positive and higher than 1 is used in the present analysis. This led to the evaluation of a mixing energy of $1.5 \mathrm{kcal} / \mathrm{mole}$. The phase diagrams of all the binary mixtures are similar and present one critical point. The miscibility of the binary mixtures at that critical point corresponds to an optimal mole fraction of 0.5 but for different temperature for each binary mixture. In fine, the polyglycolide is miscible with polylactide for all the temperature range and above $378 \mathrm{~K}$ for polyacrylonitrile, polyethylene and polystyrene. The results obtained are in agreement with those found in the literature.
\end{abstract}

Keywords: Polymers, Miscibility, Molecular Dynamics, Blends, Flory-Huggins Model, Forcite, Polyglycolide

\section{Introduction}

In recent year, increasing attention has been paid on the study of polymers because of their various applications. Sihama Issa Salih et al. [1] have studied the effect of addition different ratio from Acrylonitrile-Butadiene-Styrene (ABS) and Ethylene propylene diene monomer (EPDM) of the optimal polymer blend (PS: $5 \% \mathrm{PP}$ ) on the mechanical properties such as fracture strength, young's modulus, flexural strength, flexible modulus, maximum shear stress and hardness. Yasser M. Riyad et al [2] used azobenzene and its derivatives in solution and in polymer thin films to design polymer materials whose properties can be reversibly changed by illumination with light. The azobenzene/polymers have been shown to be interesting photoactive materials. For compliance with fire safety regulations, flame-retardant treatments of cotton in textile applications were developed by Sechin Chang et al [3] using branched polyethylenimine polymer, kaolin, urea, and diammonium phosphate. For medical application, Md. Shariful Islam and Mitsugu Todo [4] investigated the natural biocompatible and degradable biopolymer such as collagen 
and chitosan derived from chitin have been combined with the non-toxic and water-soluble synthetic polymer such as the poly (vinyl alcohol) to develop porous scaffold for tissue engineering and organ regeneration since they facilitate cell attachment and maintenance of differentiation function.

All these above-mentioned systems may be extended to polymer mixtures extending the range of chemical structure and chemical environment which impact on the switching properties of the resulting photoactive materials.

Blend polymer can lead to the development of new types of materials [5-6]. In this context, the study of miscibility between polymers is very important [7]. Generally, the goal is to produce a mixture with optimal physical and chemical properties. Current experimental methods imply the blend of polymers with different ratios and different temperature to find the adequate configuration. So, a lot of time (weeks sometimes months) is taken to find the adequate configuration. Thus, a considerable amount of time is wasted to find the optimal mixture.

Molecular dynamics simulation has been used to study properties and miscibility of a blend polymer [8-11]. Recently, simulation by molecular dynamics to predict miscibility between polymers has become very popular [12$16]$.

The Blends Module combines a modified Flory-Huggins model [17, 18] and molecular simulation techniques [19] to calculate the compatibility of binary mixtures [20].

This module allows us to analyze the material's properties, and then predicts parameters such as the interaction parameter of Flory-Huggins ( $\chi$ or Chi), the mixing energy or the phase diagrams $[17,21]$.

The Flory - Huggins parameter $\chi$ was put forth to estimate the miscibility behavior of binary mixture, for polymerpolymer mixture.

Polyglycolide is a thermoplastic and biodegradable polymer [22]. As said above, the mixture between two polymers allows us to find new stable mixture with new physical properties. Moreover, the addition of a given biodegradable polymer to a non-degradable polymer may lead to a reduction of waste in the environment. The miscibility of polymers Blends describes the homogeneity of polymer mixtures at some temperature. It can be influenced by various factors such as morphology, reduction of surface tension, crystalline and inter-molecular interaction.

In recent years, several studies of the miscibility of polymers Blends have been studied. The binary Blends miscibility of poly(3-hydroxydebutyrale) and polyethylene oxide) has been studied by YANG et al [23] they indicated that the mixture system studied is miscible. LUO et al.[24] have studied the miscibility of poly(ethylene oxide)(PEO)/poly(vinyl chloride)(PVC) Blends and they have suggested that $\mathrm{PEO} / \mathrm{PVC} 70 / 30$ and $30 / 70$ Blends are more miscible than $50 / 50$ Blend. The miscibility of chitosan(CS)/poly(ethylene oxide)(PEO) Blends was studied by RAKKAPAO et al [25] who show that miscible CS/PEO Blends were obtained only with weight fraction $\left(\mathrm{W}_{\mathrm{PEO}}\right)<0.58$. The compatibility of amphiphilic $\mathrm{pH}$-sensitive polymer
(Docosahexaenoic acid-Histidine-Lysine, DHA-His $\mathrm{Lys}_{10}$ ) and hydrophobic drug (doxorubincine, DOX) was investigated by WANG et al [26] indicating that the compatibility of the systems at $\mathrm{pH}>6.0$ is better than that at $\mathrm{pH}<6.0$. KAKHULEE et al [27] were performed a study of the miscibility of polylactic acide(PLA)/polyethylene glycol Blends and show that PLA/PEG mixtures with 90:10, 80:20 and 70:30 (wt/wt) composition are miscible. The miscibility of PLA/PLA $-\mathrm{PEG}_{\mathrm{y}}-\mathrm{PLA}_{\mathrm{x}}$ Blend was also studied by TAKHULEE et al [28]. They observed miscibility but in a smaller PEG domain in PLA/PLA $A_{x}-P G_{y}-P^{2} A_{x}$ Blends compared to PLA/PEG Blends. Wu et al [29] studied the miscibility of polyvinyl alcohol/polyvinyl pyrrolidone Blends and have seen that mixture can be miscible with any sample mass fraction by the enthalpy contributions of $\mathrm{H}$-bonds. Evaluation of compatibility in polypropylene(PP)/poly(lactic acid) (PLA)/ethylene vinyl alcohol (EVOH) ternary Blends has been studied by AMINI et al [30] they confirmed that EVOH a good compatibilzing effect of it between PP and PLA components.

In this work, a method of Forcite and Blends simulations by computer is presented. The miscibility between PGA and the following polymers: polyethylene (PE), polystyrene (PS), polyacrylonitrile (PAN) and polylactide (PLA) is analyzed by calculating the interaction parameter of Flory-Huggins, the energy mixing and phase diagrams. In addition, the blending temperatures were measured. In view of their use in the electrospin of nanofibers.

\section{Simulation Methods}

Forcite and Blends modules from Materials Studio 2017 were used for the different simulations.

\subsection{Forcite Simulation}

Forcite is a molecular mechanics module for potential energy and geometry optimization calculations of arbitrary molecular and periodic systems using classical mechanics.

Optimization is used to bring about the most stable configuration of a constructed structure.

The Dreiding [31] force fields were employed in this study. The 'Dreinding' force field represents the intra- and intermolecular interactions.

Structural optimization is a two-steps process: energy evaluations and conformation adjustment.

The coordinates of a structure combined with a force field create an energy expression (or a target function). This energy expression is the equation that describes the potential energy of a particular structure as a function of its atomic coordinates.

The total potential energy $\left(E_{p o t}\right)$ of the system is expressed:

$$
E_{\text {pot }}=E_{\text {valence }}+E_{\text {Crossterm }}+E_{\text {non-bond }}
$$

The energy of valence interaction $\left(\mathrm{E}_{\mathrm{valence}}\right)$ includes the stretching energy of the bond $\left(\mathrm{E}_{\mathrm{bond}}\right)$, the bending energy of the valence angle $\left(E_{\text {angle }}\right)$, the torsion energy of the dihedral 
angle $\left(\mathrm{E}_{\mathrm{torsion}}\right)$ and the inversion energy called out-of-plane interaction (oop), which are part of nearly all forcefields for covalent systems. Cross-term energy ( $\left.E_{\text {crossterm }}\right)$ has been used to account for such factors as bond or angle distortions caused by nearby atoms. These terms are necessary to reproduce with precision the vibrational frequencies and therefore, the dynamic properties of molecules. Finally, the energy of interactions between non-bonded atoms ( $\left.E_{\text {non-bond }}\right)$ includes the van der Waals energy $\left(E_{\mathrm{vdw}}\right)$, hydrogen bond energy $\left(\mathrm{E}_{\mathrm{H} \text {-bond }}\right)$ and Coulomb electrostatic energy $\left(\mathrm{E}_{\mathrm{Coulomb}}\right)$.

The conformation is adjusted to reduce the value of the energy expression. A minimum may be found after one adjustment or may require several thousand of iteration, depending on the nature of the algorithm, the form of the energy expression and the size of the structure. The efficiency of optimization is therefore judged both by the time required to evaluate the expression of energy and the number of structure adjustment (iterations) necessary to converge to a minimum.

A geometric optimization is first done for each sample (glycolide with one of the following lactide, acrylonitrile ethylene and styrene) monomers. With glycolide monomer mixed successively with one of the four monomers (lactide, acrylonitrile ethylene and styrene). The Forcite module is used for this optimization. Each sample was subjected to 50 000 steps of energy minimization with the convergence tolerance for energy and force are $1.10^{-4} \mathrm{kcal} / \mathrm{mol}$, $0.005 \mathrm{kcal} / \mathrm{mol}$ respectively for a maximum iteration equal to 50. The forcefield type is Dreiding, and the approach used for calculating the atomic charge is based on the "charge-using QEq" method. The convergence limit was fixed at $5.10^{-4} \mathrm{e}$ for 50 maximum iterations. The energy bin width was set to $0.2 \mathrm{kcal} / \mathrm{mol}$, and the atom-base summation was applied for both Electrostatic and Van der Waals interaction with a cutoff distance of $18.5 \AA$, a spline width of $1 \AA$ and a $0.5 \AA$ buffer width [32].

\subsection{Blends Simulation}

The blends module in Materials Studio has been developed to study the miscibility of polyglycolide and the following polymers: polyethylene, polystyrene, polyacrylonitrile and polylactide, greatly reducing the need for laboratory experimentation. Analysis of the results to predict the miscibility, such as Flory-Huggins $\chi$ parameter, phase diagrams and binding energies for two components. This module has a unique superiority and a high efficiency. This method can predict the thermodynamics of the mixing directly from the chemical structures of two components and therefore only requires their molecular structures and a forcefield as input to the Blends simulation. Its unique superiority lies on the fact that it combines a modified FloryHuggins model [17, 18] and a molecular simulation technique [19] to calculate the compatibility of polymerpolymer mixtures.

Two important extensions of the Flory-Huggins model are used:

(i) Blends incorporates an explicit temperature dependence on the interaction parameter. This is accomplished by generating a large number of pair configurations and calculating the binding energies, followed by temperature averaging the results using the Boltzmann factor and calculating the temperaturedependent interaction parameter.

(ii) Blends is an off-lattice calculation, meaning that molecules are not arranged on a regular lattice as in the original Flory-Huggins theory. The coordination number is explicitly calculated for each of the possible molecular pairs using molecular simulations.

These two extensions of the classical Flory-Huggins theory of mixing are documented in publications by Blanco [21] and Fan et al [19].

The Flory-Huggins model used in the binary system is at the basis of the thermodynamics of mixing, in the polymer / polymer and solvent / polymer systems [20]. This is the simplest and most widely used approach for calculating the free energy of the mixture and for phase diagram construction of a binary system. The general expression for the free energy of a mixing of a binary system is:

$$
\frac{\Delta G}{R T}=\frac{\phi_{A}}{n_{A}} \ln \phi_{A}+\frac{\phi_{B}}{n_{B}} \ln \phi_{B}+\chi \phi_{A} \phi_{B}
$$

Where $\Delta G$ is the free energy of mixing (per mol), $\phi_{i}$ is the volume fraction of component $\mathrm{i}, \mathrm{n}_{\mathrm{i}}$ is the degree of polymerization of component i, $\chi$ is the interaction parameter, $\mathrm{T}$ is the absolute temperature, and $\mathrm{R}$ is the gas constant.

The first two terms represent the combinatorial entropy. This contribution is always negative, hence favoring a mixed state over the pure components. The last term is the free energy due to interaction. If the interaction parameter, $\chi$, is positive, this term disfavors a mixed state. The balance between the two contributions gives rise to various phase diagrams.

The Flory-Huggins interaction parameter, $\chi$, is defined as:

$$
\chi=\frac{E_{m i x}}{R T}
$$

Where $\mathrm{E}_{\mathrm{mix}}$ is the mixing energy; that is, the difference in free energy due to interaction between the mixed and the pure state. In the traditional Flory-Huggins model, each component occupies a lattice site. For a lattice with coordination number $\mathrm{Z}$, the mixing energy is:

$$
E_{m i x}=\frac{1}{2} Z\left(E_{b s}+E_{s b}-E_{b b}-E_{s s}\right)
$$

Where Eij is the binding energy between a unit of component $\mathrm{i}$ and a unit of component $\mathrm{j}$. For molecules, the binding energies have to be regarded as averages over an ensemble of molecular configurations. In the extended FloryHuggins model, these degrees of freedom are incorporated.

Temperature effects can be taken into account, however, by weighting the distribution with the Boltzmann factor, 
$\exp \left(-\frac{E_{i j}}{R T}\right)$. The average binding energy at temperature $\mathrm{T}$ is the average of the weighted distribution function:

$$
\left\langle E_{i j}\right\rangle_{T}=\frac{\int d E E P_{i j}(E) e^{-E / R T}}{\int d E P_{i j}(E) e^{-E / R T}}
$$

Where $P_{i j}$ is the distribution energy.

After having determined all four average binding energies as a function of the temperature and the coordination numbers, the interaction parameter $\chi$ at the particular temperature can be determined from the more general equation. 6 or equation. 4 .

Once the temperature-dependent average binding energies and the average coordination numbers are calculated, it is easy to determine the mixing energy at a temperature $\mathrm{T}$ :

$$
E_{m i x}=\frac{1}{2}\left(Z_{b s}\left\langle E_{b s}\right\rangle_{T}+Z_{s b}\left\langle E_{s b}\right\rangle_{T}-Z_{b b}\left\langle E_{b b}\right\rangle_{T}-Z_{s s}\left\langle E_{s s}\right\rangle_{T}\right)
$$

The interaction parameter, $\chi$, is simply the mixing energy divided by RT, following Eq.3. $\chi$ is the central quantity in the Flory-Huggins theory. Its temperature dependence gives rise to various phase diagrams.
The binding energy is a measure of the energy of interaction between the two components. Together with the coordination numbers, it enables generation of the mixing energy and the $\chi$ parameter and of phase diagrams. Blends generates a large number of molecular orientations and calculation the of pair interaction energies of each configuration has to be done.

In evaluating the blinding energies, Blends distinguishes the components by using the property role: on component has a base role, the other has a screen role. A given base-screen combination can give four potentially different pairs, each of which will have an associated binding energy value: [21]

(i) Base-base pair $\left(\mathrm{E}_{\mathrm{bb}}\right)$

(ii) Screen-screen pair $\left(\mathrm{E}_{\mathrm{ss}}\right)$

(iii) Base-screen pair $\left(\mathrm{E}_{\mathrm{bs}}\right)$

(iv) Screen-base pair $\left(\mathrm{E}_{\mathrm{sb}}\right)$

The last two pair are equivalent. Blends only calculates the energy of a base-screen pair and uses this value for the energy of a screen-base pair.

The glycolide, lactide, acrylonitrile, ethylene and styrene monomers units used in our blends simulation to determine the head and tail atoms are shown in figure 1. The geometries of these repeat units monomer are optimized by Forcite Module, before submitting for the blend calculation.

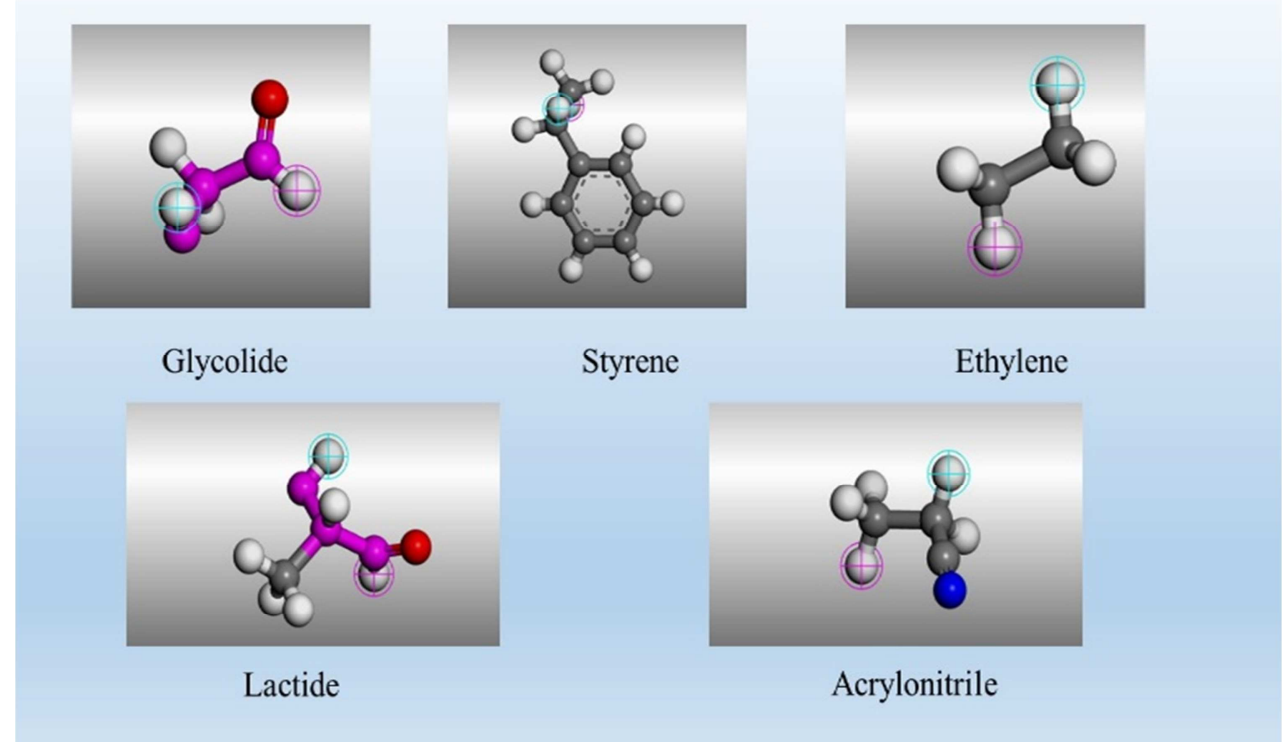

Figure 1. The monomer repeat unit structure models of glycolide, styrene, ethylene, lactide and acrylonitrile.

The blends button on the menu toolbar is selected on dropdown menu and then the calculation is selected from the dropdown list of the module. In the input section of the setup tab, the empty part named Molecule must be selected to insert the molecules that have been optimized by Forcite. On the setup tab of the Blends calculation dialog, the accuracy has been changed from medium to fine corresponding respectively to a bin width of $0.2 \mathrm{kcal} / \mathrm{mol}$ to $0.02 \mathrm{kcal} / \mathrm{mol}$. Then, on the same dialog box (Blends Calculation) the Energy tab is selected. The force field is set to Dreiding and changed the 'charge to charge' using 'QEq' for the computation of Flory-Huggins interactions.

After the previous steps, on the Blends Calculation dialog, the Run option is selected. A new file, of glycolide mixture is created in the Project Explorer. After the simulation, the Blend Analysis Module is selected from the menu bar to open the Blend Analysis dialog. The analysis option, allows us to study the mixing energy, the $\chi$ parameter and the phase diagrams. The blends module of the system is validated by the calculation made by Fei-Zhou Li and al [32]. 


\section{Results and Discussion}

\subsection{Parameter of Flory-Huggins}

The Flory-Huggins parameter $\chi$ was put forward to estimate the miscibility behavior of the binary mixtures, such as polymer-polymer, solvent-solvent and polymer-solvent. Thus, $\chi$ could also be used to describe the miscibility of component in systems. According to the Flory-Huggins theory, a negative value or a value less than 1 of $\chi$ indicates that at this particular temperature the two molecules have better miscibility and a favorable interaction. It is likely that at this temperature a mixture of the two components will show just one phase. If $\chi$ is large and positive, the molecules both prefer to be surrounded by similar components rather than each other and cannot be mixed [19]. If the value of $\chi$ is very large, this contribution to the free energy overcomes the combinatorial entropy and a mixture of the two components will separate into two different phases.

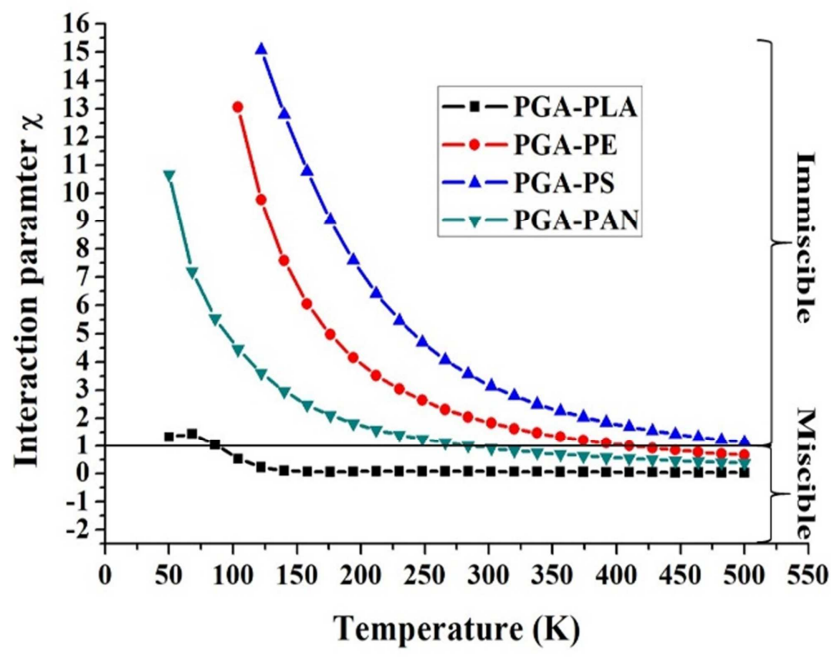

Figure 2. $\chi$ Interaction parameters as a function of the temperature for the binary mixing systems of PGA/PLA, PGA/PE, PGA/PS and PGA/PAN.

In figure 2 , the interaction parameter $\chi$ is plotted as a function of the temperature, for mixture of polyglycolide with polylactide, polystyrene, polyethylene and polyacrylonitrile.

As seen from Figure 2, for the mixing PGA with PS, PE or PAN, when the temperature increases, the $\chi$ parameter decreases and remains always positive, while $\chi$ parameter the PGA with PLA can mix for all the temperature range. However, miscibility of the mixing system for PGA with PAN, PE and PS only acceptable at temperature $230 \mathrm{~K}, 238 \mathrm{~K}$ and $378 \mathrm{~K}$ respectively. The miscibility for Blends PGA with PLA were better. At higher temperatures, systems for all Blends indicated excellent miscibility. Analysis of the results in Figure 2 showed that PGA with PLA and PAN had the best miscibility, followed by PE; PGA with PS had the lowest miscibility. In other words, PGA showed optimal miscibility with polymers superior than $378 \mathrm{~K}$.

\subsection{Mixing Energy}

In addition to $\chi$ parameter, it is generally admitted that a value of the mixing energy $\left(\mathrm{E}_{\text {mix }}\right)$ close to zero shows miscibility. More $\mathrm{E}_{\mathrm{mix}}$ increases, less the polymers are miscible.

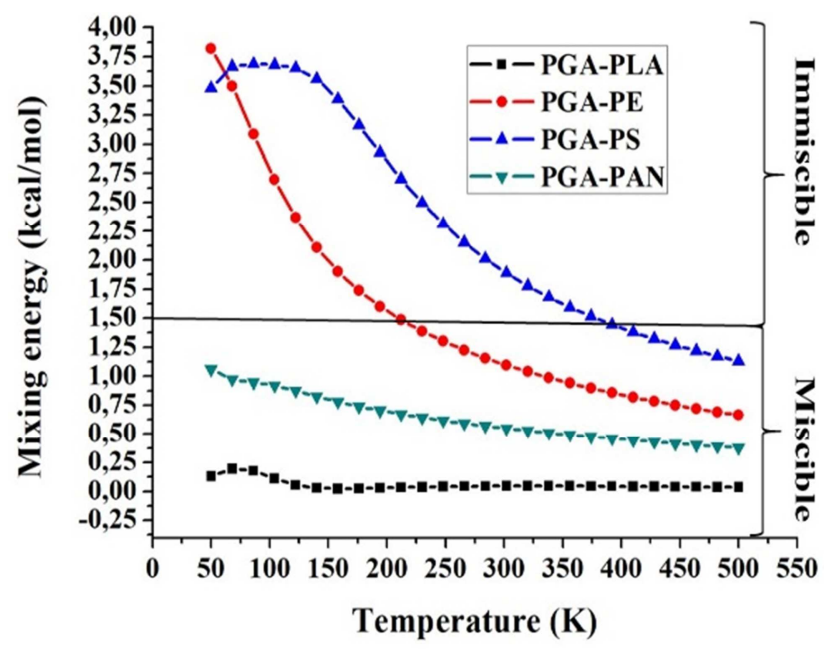

Figure 3. Mixture energy as a function of temperature for binary mixing systems of PGA/PLA, PGA/PE, PGA/PS and PGA/PAN.

Then, in figure $3 \mathrm{E}_{\mathrm{mix}}$ is plotted as a function of the temperature, for mixtures of PGA with PLA, PAN, PE and PS. The analysis of the graph shows that when the temperature increases, the energies of mixtures PGA with PLA, PAN and PE decrease, while for mixing PGA with PS has not changed significantly, but his $\mathrm{E}_{\text {mix }}$ increases to $104 \mathrm{~K}$ before reducing; when the temperature is superior than $378 \mathrm{~K}$, the mixing energy value for all mixtures are close to 1 . Thus, the results reinforce the conclusions of the interaction parameter $\chi$, the PGA will be miscible with PLA, PAN, PE and PS at a temperature superior $378 \mathrm{~K}$.

\subsection{Phase Diagrams of the Binary Mixture}

Phase diagrams are very useful in illustrating the compatibility of binary mixtures $[18,19]$, which is derived from the free energy of mixture. They give information in which temperature range the mixture is unstable and at which temperature the mixture is miscible. An unstable binary mixture will separate in two phase, the compositions for which can be read from the diagram. It generally contains three pieces of information: on critical points (red), spinodal (blue) and bimodal (black). Using Blends simulation, the results of phase diagram for PGA with other polymers (PLA, PAN, PE and PS) are represented in Figure 4. In phase diagrams, a critical point marks the start of coexistence region. In the coexistence region, the mixture can lower its free energy by separating into two phases. In Figure 4, all mixtures have a simple critique point and one coexistence region. However, more complex mixtures may have more than one critical point and several regions of coexistence. The coexistence region is linked by the binodal (black lines in Figure 4). Meanwhile, the spinodal (blue lines in Figure 4) separates the coexistence region into two regions. In the region between the binodal and 
the spinodal (the region between the blue and black lines of Figure 4) the mixture is metastable, in the sense that the mixture will begin to separate only after a sufficiently large fluctuation. But in the region bounded by the spinodal (the region situated between the blue lines of Figure 4) the mixture is unstable; any fluctuation will cause the spontaneous separation of the mixture. Likewise, the maximum of the spinodal corresponds to the critical point. In other words, the mixture is stable above the binodal region.
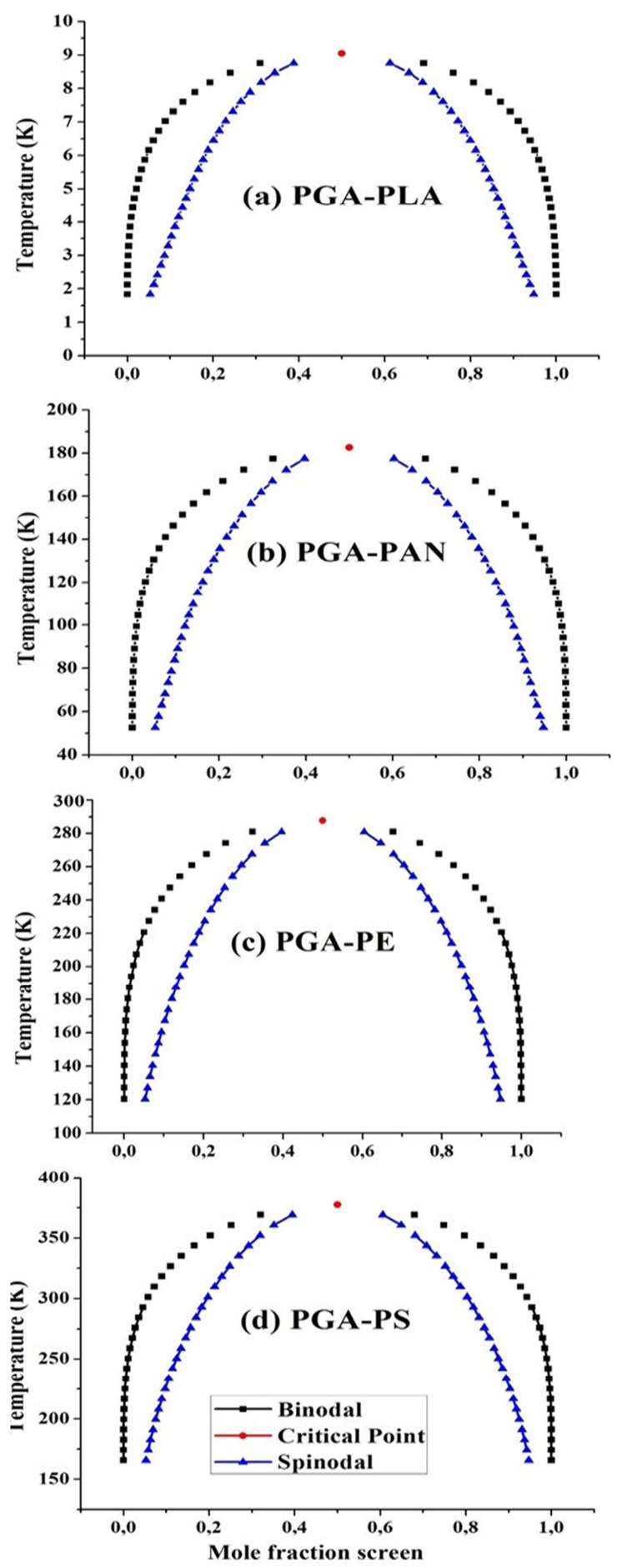

Figure 4. Phase diagrams for binary systems compound of: (a) PGA/PLA, (c) PGA/PAN, (b) PGA/PE and (d) PGA/PS.
Thee phase diagrams of PGA with other polymers (PLA, PAN, PE and PS) calculated by Blends are shown in Figure 4, where the composition of the mixture is given as a function of the mole fraction screen of the other polymers (PLA, PAN, PE and PS). The results have shown that the temperature of the critical point for PGA/PLA, PGA/PAN, PGA/PE and PGA/PS are $9 \mathrm{~K}, 183 \mathrm{~K}, 288 \mathrm{~K}$ and $378 \mathrm{~K}$ respectively. It is also stated that PGA was mixed with different polymers above the critical point as the reference temperature for mixing with the polymers. In addition, an optimum mole fraction of 0.5 for all the mixture was found.

The results of the phase diagrams are in agreement with the previous results of $\chi$ and $\mathrm{E}_{\mathrm{mix}}$.

\section{Conclusions}

In this study, the state of miscibility between the PGA blends with the PLA, PAN, PE and PS by molecular dynamics simulation was examined. The Flory-Huggins interaction parameter, mixing energy and phase diagrams of blends systems for PGA with PLA, PAN, PE and PS were constructed. The results showed that PGA with PLA was miscible in all the temperature range. Moreover, at a temperature of about $378 \mathrm{~K}$, PGA is miscible with all other polymers (PAN, PE and PS). Likewise, an optimal mole fraction is find at 0.5 . Then, we can say that for a mole fraction of 0.5 and a temperature of $378 \mathrm{~K}$ PGA is miscible with all other polymers (PLA or PAN or PE or PS). In perspective, nanofiber of these blends polymer will be made by electrospinning deposition.

\section{References}

[1] Sihama Essa Salih, Jawad Kadhum Oleiwi, Rawaa Adnan Abdle Ameer. Evaluation of Addition ABS and EPDM Effect on the Mechanical Properties of Ternary Polymer Blends. International Journal of Materials Science and Applications. Vol. 4, No. 1, 2015, pp. 39-46.

[2] RIYAD, Yasser M., NAUMOV, Sergej, GRIEBEL, Jan, et al. Optical switching of azophenol derivatives in solution and in polymer thin films: The role of chemical substitution and environment. Am. J. Nano Res. Appl, 2014, vol. 2, p. 39-52.

[3] Sechin Chang, Brian Condon, Elena Graves, Jade Smith. AntiFlammable Properties of Cotton Fabrics Using Eco Friendly Inorganic Materials by Layering Self-Assisted Processing. International Journal of Materials Science and Applications Vol. 7, No. 4, 2018, pp. 115-125.

[4] Md. Shariful Islam, Mitsugu Todo. Improved Mechanical Properties of PVA-Chitosan Polymeric Porous Scaffolds for Tissue Engineering. American Journal of Clinical and Experimental Medicine. Vol. 3, No. 5, 2015, pp. 268-274.

[5] UTRACKI, Leszek A. Compatibilization of polymer blends. The Canadian journal of chemical Engineering, 2002, vol. 80, no 6, p. 1008-1016.

[6] Inger M. A., Emilio M., Borja C. Polymer, 51 (2010) 44314438 . 
[7] SINGH, Y. P. and SINGH, R. P. Compatibility studies on solutions of polymer blends by viscometric and ultrasonic. European Polymer Journal, 1983, vol. 19, no 6, p. 535541.

[8] Tiller A. R., Gorella B. Polymer, 35 (1994) 32-51.

[9] Choi P., Blom H. P., Kavassalis T. A., Rudin A. Macromolecules, 28 (1995) 82-47.

[10] Choi K., Jo W. H. Macromolecules, 31 (1998) 13-66.

[11] Lee S., Lee J. G., Lee H., Mumby S. Polymer, 40 (1999) 5137.

[12] Hua Y., L. S., Qian H. J. Polymer, 45 (2004) 453-457.

[13] Jawalkar S. S., Aminabhavi T. M. Polymer, 47 (2006) 80618071.

[14] DE ARENAZA, Inger Martinez, MEAURIO, Emilio, COTO, Borja, and al. Molecular dynamics modelling for the analysis and prediction of miscibility in polylactide/polyvinilphenol blends. Polymer, 2010, vol. 51, no 19, p. 4431-4438.

[15] FU, Yizheng, LIAO, Liqiong, LAN, Yanhua, and al. Molecular dynamics and mesoscopic dynamics simulations for prediction of miscibility in polypropylene/polyamide-11 blends. Journal of Molecular Structure, 2012, vol. 1012, p. 113-118.

[16] FU, Yizheng, LIAO, Liqiong, YANG, Luxia, and al. Molecular dynamics and dissipative particle dynamics simulations for prediction of miscibility in polyethylene terephthalate/polylactide blends. Molecular simulation, 2013, vol. 39 , no 5, p. 415-422.

[17] K. F. Freed, J. Phys. A: Math. Theor., 1985, 18, 871.

[18] K. S. Schweizer and J. G. Curro, J. Chem. Phys., 1989, 91, 5059 .

[19] C. F. Fan, B. D. Olafson. and M. Blanco, Macromolecules, 1992, 25, 3667-3676.

[20] Flory, Paul J. Principles of polymer chemistry. Cornell University Press, 1953.

[21] BLANCO, Mario. Molecular silverware. I. General solutions to excluded volume constrained problems. Journal of computational chemistry, 1991, vol. 12, no 2, p. 237.

[22] NAIR, Lakshmi S. et LAURENCIN, Cato T. Biodegradable polymers as biomaterials. Progress in polymer science, 2007, vol. 32 , no $8-9$, p. $762-798$.
[23] YANG, Hua, ZE-SHENG, Li, QIAN, Hu- jun, et al. Molecular dynamics simulation studies of binary blend miscibility of poly (3-hydroxybutyrate) and poly (ethylene oxide). Polymer, 2004, vol. 45, no 2, p. 453-457.

[24] LUO, Zhonglin et JIANG, Jianwen. Molecular dynamics and dissipative particle dynamics simulations for the miscibility of poly (ethylene oxide)/poly (vinyl chloride) blends. Polymer, 2010, vol. 51, no 1, p. 291-299.

[25] RAKKAPAO, Natthida et VAO-SOONGNERN, Visit. Molecular simulation and experimental studies of the miscibility of chitosan/poly (ethylene oxide) blends. Journal of Polymer Research, 2014, vol. 21, no 12, p. 606.

[26] WANG, Yan, REN, Jia Wei, ZHANG, Can Yang, et al. Compatibility studies between an amphiphilic $\mathrm{pH}$ - sensitive polymer and hydrophobic drug using multiscale simulations. RSC Advances, 2016, vol. 6, no 103, p. 101323-101333.

[27] TAKHULEE, Adisak, TAKAHASHI, Yoshiaki, et VAOSOONGNERN, Visit. Molecular simulation and experimental studies of the miscibility of polylactic acid/polyethylene glycol blends. Journal of Polymer Research, 2017, vol. 24, no 1, p. 8.

[28] TAKHULEE, Adisak, TAKAHASHI, Yoshiaki, et VAOSOONGNERN, Visit. Molecular simulation and xperimental studies of the miscibility of PLA/PLAx-PEGy-PLAx blends. Journal of Polymer Research, 2017, vol. 24, no 11, p. 178.

[29] WU, Hui et XIN, Yong. Molecular dynamics and MesoDyn simulations for the miscibility of polyvinyl alcohol/polyvinyl pyrrolidone blends. Plastics, Rubber and Composites, 2017, vol. 46 , no 2 , p. 69-76.

[30] AMINI, M., MOBLI, M., KHALILI, M., et al. Assessment of Compatibility in Polypropylene/Poly(lactic acid)/Ethylene vinyl alcohol Ternary Blends: Relating Experiments and Molecular Dynamics Simulation Results. Journal of Macromolecular Science, Part B, 2018, no just-accepted, p. 131.

[31] Mayo, S. L., Olafson, B. D., Goddard, W. A., 1990. III DREIDING: A generic forcefield for molecular simulations. Journal of Physical Chemistry 94, 8897-8909.

[32] LI, Fei-Zhou, LU, Zhen-Lin, and TIAN, Dong-Ping. A Combined Experimental and Molecular Dynamics Simulation Study on the Miscibility of Eucommia Ulmoides Gum with Several Rubbers. Polymers \& Polymer Composites, 2017, vol. 25 , no 1, p. 87. 